\title{
Fire resistance evaluation of simple steel hall carried out by using various computational models
}

\author{
M. Maślak, M. Pazdanowski, M. Suchodoła \& P. Woźniczka \\ Cracow University of Technology, Cracow, Poland
}

\begin{abstract}
It has been shown, how the selection of static scheme for a single transverse frame in a typical steel hall determines the sought fire resistance of that hall. For comparative purposes several computational models differing in complexity have been considered for the same fire development scenario and the same frame. Loss of the capacity to safely resist the applied static loads, identified on the equilibrium path, has been considered as the representative measure of resistance against increasing in fire temperature of structural steel components. Juxtaposition and comparison of the obtained results leads to the unequivocal conclusion, that the higher the level of simplification in the applied computational model, the more overestimated the resultant fire resistance determined based on that model.
\end{abstract}

\section{INTRODUCTION}

In order to obtain a reliable fire resistance estimate for a steel hall, one should perform the analysis referring to the 3D bearing structure treated as a whole, or, if the available computer resources exclude this option, determine this resistance approximately, after detailed analysis of a single transverse frame formally isolated from structural system. The time during which such a frame will be able to safely resist the loads applied to it under the same fire development scenario accompanied by progressing material weakening will differ depending on the model selected to represent the considered structure. In general the fire resistance forecast for a steel frame is determined numerically, based on a bar model of all structural components, subject to the assumption that the temperature of these components is evened out not only in the respective cross sections but also along their whole lengths. In this analysis we intend to show how the selection of a particular static scheme affects the sought fire resistance. In order to do that a selected typical one-aisle and one-storey high steel hall is subjected to a simulated fire action by using various computational models. The calculations have been executed using SAFIR computer code (Franssen \& Gernay, 2017). In each considered case the final fire resistance, specified for the same frame, but modelled in a different way, has been identified on the static equilibrium path corresponding to that model.

\section{DESCRIPTION OF THE ANALYZED FRAME AND CHARACTERISTICS OF THE CONSIDERED COMPUTATIONAL MODELS}

A single transverse frame of a typical steel hall having the geometry, dimensions, rafter and column cross sections depicted in Figure la has been selected as the basis for comparative analysis. All the structural components of the frame have been made of S235 steel. It is assumed, that fully developed fire has been initiated inside the hall, such that during every moment of the fire action the temperature of combustion plume has been evened out in the

DOI: $10.1201 / 9781003132134-66$ 
a)

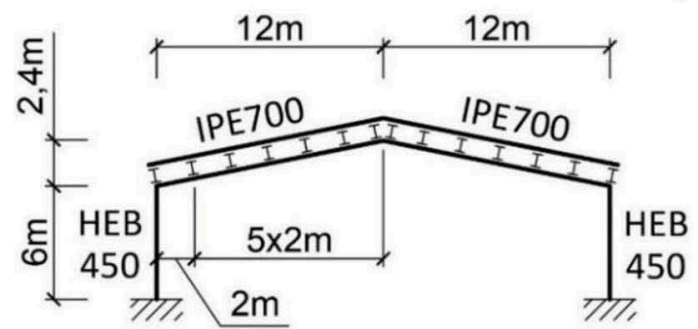

b)

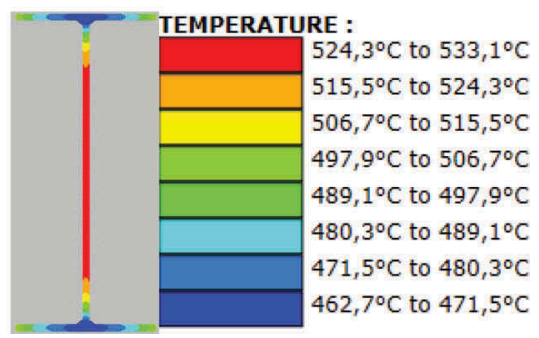

Figure 1. The reference frame: a) geometry and a static scheme, b) steel temperature distribution in the rafter identified after 660 seconds of fire exposure.

whole fire compartment and increased in time following the classical scenario of the standard fire. Due to the different ratios of heated periphery to the surface area of a cross-section, specified for the thermally uninsulated frame components, those components have been heated with different speeds. The scheme of uniform fire exposure of such the components along the entire perimeter of their cross-section has been selected for the analysis (i.e. their heating from four sides). In the authors' opinion, considering the case of heating these elements from only three sides, which could be selected as an alternative analysis, in the case of a hall with a light housing would not lead to a more precise and more accurate description of the reality. As a result of such the findings the uneven distribution of steel temperature field in the cross section of columns and rafter in each moment of fire has been accounted for, though under assumption, that this distribution does not change along their lengths. The characteristics of particular thermal actions related to selected moments of fire are shown in the Table 1. This table is accompanied by a sample map of temperature distribution in a rafter, obtained after 660 seconds of fire exposure (Figure 1b). It is assumed, that both left and right end plate beam to column joints remain fully rigid during the whole time of fire.

The transverse frame described above subjected to numerically simulated fire exposure of intensity increasing in time in the following analysis is modelled with application of several computational models differing in complexity. These models are listed in turn below:

- models A1 and A2 - understood as fully 2D models. Only deformations occurring in the frame plane are accounted for in these models. Flexural in plane buckling of rafter or column, i.e. the global instability of an element about the "stronger" axis of its cross section, usually determines the bearing capacity here. Model A1 pertains to the frame with fully rigid supports, while model A2 pertains to the analogous frame with pinned supports.

- Model B1 - frame with rigid supports. An out of frame plane deformation, including classical flexural out of plane buckling as well as lateral torsional buckling of column or rafter, is accounted for. Thus the bearing capacity may be determined by the global instability of an element about the "weaker" axis of its cross section. Such a scheme of transverse braces in the hall roof has been assumed, as to assure that every other purlin would constitute a support stabilizing the rafter in the frame plane at its point of support (Figure 2).

Table 1. Steel temperature in the rafter and columns of the considered frame after various fire exposure times (its minimum and maximum values identified at the cross-section heights).

\begin{tabular}{llll}
\hline \multirow{2}{*}{ Fire duration $[\mathrm{s}]$} & \multicolumn{2}{l}{ Temperature $\min / \max \left[{ }^{\circ} \mathrm{C}\right]$} \\
\cline { 2 - 4 } & Fire plume $\Theta_{g}$ & Rafter $\left(\right.$ IPE700) $\Theta_{a}^{\text {beam }}$ & Columns $\left(\right.$ HEB450) $\Theta_{a}^{\text {column }}$ \\
\hline 180 & 502 & $104 / 135$ & $77 / 128$ \\
360 & 603 & $240 / 300$ & $179 / 286$ \\
540 & 663 & $380 / 452$ & $292 / 434$ \\
720 & 705 & $499 / 567$ & $402 / 516$ \\
900 & 738 & $591 / 647$ & $498 / 633$ \\
\hline
\end{tabular}



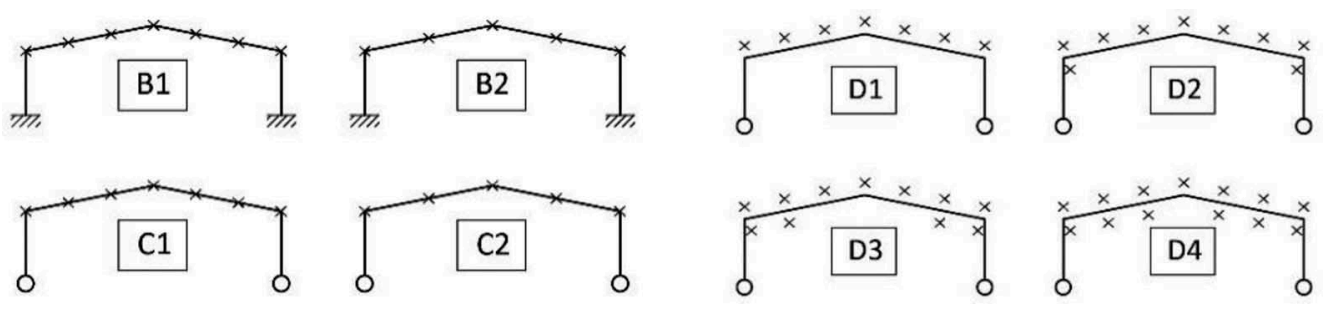

Figure 2. Computational models considered in this paper.

- Model B2 - analogous to the model B1, but in this case, due to the different distribution of braces in the roof plane only every third purlin stabilizes the rafter (Figure 2). Frame with rigid supports.

- Model C1 - analogous to the model B1, but prepared for the frame with pinned supports.

- Model C2 - analogous to the model B2, but prepared for the frame with pinned supports.

- Model C3 - analogous to the model C1, but prepared for the frame with columns and rafter made of S355 steel.

- Model D1 - analogous to the model C1, but additionally taking into account the offset of the axis of purlins bracing the rafter, identified in relation to the axis of such a rafter (Figure 2). The magnitude of this offset has been approximately assumed at $35 \mathrm{~cm}$, i.e. half of the rafter height.

- Models D2, D3 and D4 - analogous to the model D1, but taking into account an additional offset introduced by application of additional anti-twist braces in the selected cross sections of the frame. The magnitude of this offset is assumed to be equal to $35 \mathrm{~cm}$ at both sides.

In each of the considered models a nonlinear relationship between stresses and strains of the structural steel exposed to the fire action is taken into account, as well as an appropriate reduction of the steel yield limit induced by the ambient temperature increasing during fire.

\section{COMPARATIVE ANALYSIS OF THE OBTAINED RESULTS}

In each of the considered cases during the initial phase of fire exposure, due to the thermal expansion of steel, the expanding columns raised the roof ridge, and at the same time the expanding rafter pushed the columns outwards. With the progress of time the rafter, increasingly weakened by the increasing ambient temperature, exhibited increasing deflection. This deflection effectively negated the initial push up of the roof ridge. As a result the columns initially pushed in the outwards direction now became pulled inwards due to the ever increasing rafter sagging induced by the increasing fire intensity. This change in the sense of displacements in general occurs abruptly. This allows for unequivocal indication of fire exposure related to the fire resistance of the considered frame.

Detailed analysis of the equilibrium paths depicted in Figures $3 \mathrm{a}$ and $3 \mathrm{~b}$ leads to the following estimates of the fire resistance $\tau_{f i, R}[\mathrm{~s}]$ sought: for model A1 $-\tau_{f i, R}=1088 \mathrm{~s}$, for model B1 $\tau_{f i, R}=997 \mathrm{~s}$, for model B2 $-\tau_{f i, R}=899 \mathrm{~s}$. However small in absolute terms, the quantitative differences identified in this juxtaposition seem to be significant when expressed in relative terms. It has to be emphasized, that in the model A1 the frame is destroyed by the flexural in plane buckling of column or rafter, while in the models B1 and B2 the destruction occurs usually due to flexural out of plane or more probably by lateral torsional buckling. The much lower fire resistance of the frame B2 with respect to the analogous result obtained for the same frame modeled as B1 may be attributed to the larger distance between supports stabilizing the rafter in frame plane.

In Figure 4a the results analogous to those shown in Figure 3a are depicted, but obtained for the frame with pinned supports (models $\mathrm{C} 1$ and $\mathrm{C} 2$ ). This figure is juxtaposed with Figure $4 \mathrm{~b}$, to show the differences in the estimated fire resistance in the case of the same frame, but 
a)

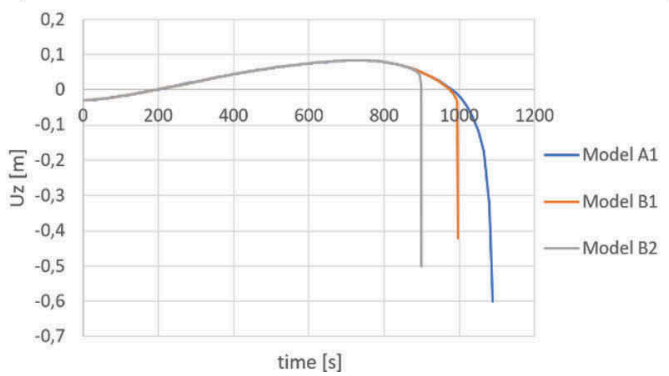

b)

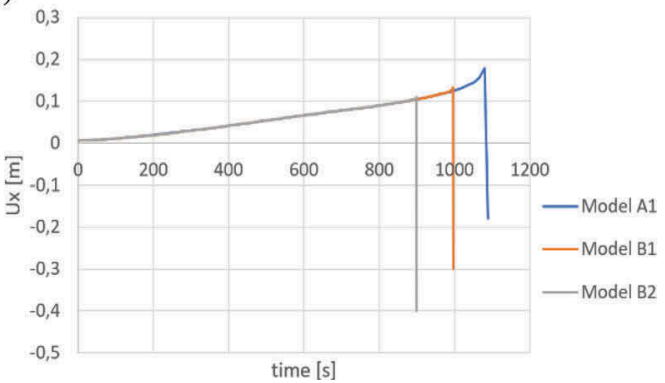

Figure 3. Static equilibrium paths obtained for the frame with fully rigid supports, for: a) roof ridge (vertical displacements, b) eaves (horizontal displacements).

a)

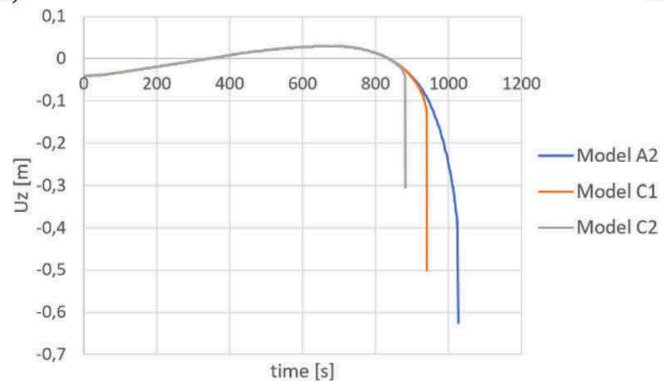

b)

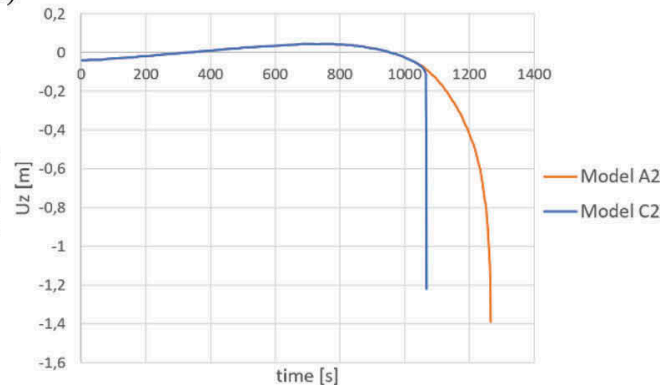

Figure 4. Static equilibrium paths related to vertical displacements for the roof ridge of a frame with pinned supports, rafter and columns made of: a) S235 steel, b) S355 steel.

this time with rafter and columns made of S355 steel, exhibiting much higher strength. The fire resistance obtained for this frame is obviously much higher.

The subsequent group of analyzed frames relates to the structure having pinned supports and modelled offsets of various origins (models D1, D2, D3 and D4). The model D1 accounts for the offset of purlin with respect to the rafter, while models D2, D3 and D4 additionally account for application of anti-twist braces in the several cross sections of the frame (Figure 2). The static equilibrium paths obtained numerically under such boundary conditions are juxtaposed in Figure 5. The fire resistance obtained after application of the D1 model proved to be much lower than the one obtained previously after application of the model $\mathrm{C} 1$. This is an adverse result of the additional moment twisting the rafter. Application of appropriate anti-twist braces efficiently eliminates this influence. In the model D1 this occurs to the relatively low degree, as the braces have been applied only in the line of columns. The stiffening effect, observed on the models D3 and D4 with much higher number of braces, is much more pronounced and therefore in those cases the fire resistance substantially exceeds the one determined previously on the model C1.

\section{CREDIBILITY OF THE MODEL WITH SINGLE TRANSVERSE FRAME}

The analysis presented above seems to indicate unequivocally, that appropriate modelling of the boundary conditions has, in the case of application of simple computational model with single transverse frame, a direct and significant influence on the obtained value of fire resistance sought. The general rule seems to be, that the higher the degree of model simplification the more overestimated the final fire resistance is with respect to the real value. Thus the 


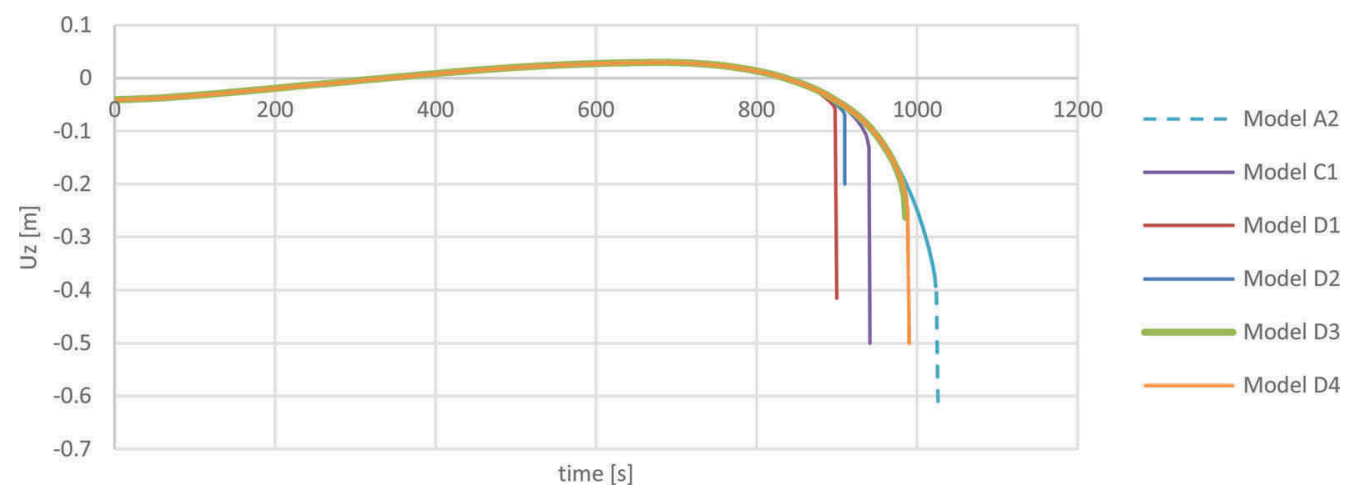

Figure 5. Static equilibrium paths related to vertical displacements obtained for the roof ridge of a frame with pinned supports, when taking into account offsets of various types (models D1, D2, D3 and D4 of Figure 2).

desire to simplify the calculations in this situation seems to be burdened by high risk, as the result obtained by the simulation may impart a completely unjustified sense of false security upon the hall user. However, the basic question remains, how credible the result of even the most accurate estimate may be to the user, if this result is supported only by a simple modelling with application of a single transverse frame. The response, to be authoritative, requires at least a comparison of static equilibrium paths obtained for the same hall and determined with application of a full 3D model taking into account the spatial interactions between structural components and a reduced 2D model consisting of a single transverse frame formally isolated from the whole structure and with relatively carefully applied boundary conditions. The results of such comparison are depicted in Figures 6a, 6b and 6c (Wozniczka, 2020). A single frame with truss rafter depicted in Figure $6 \mathrm{a}$ is in this case denoted as model E. The action of purlins and wall girts is in this model accounted for by application of appropriate supports. This simple model has been subsequently correlated with a full $3 \mathrm{D}$ model depicted in Figure $6 \mathrm{~b}$ and denoted as model $\mathrm{F}$. The static equilibrium paths obtained for both models are juxtaposed in Figure 6c. It is clearly visible, that the equilibrium path obtained in this juxtaposition for a complex model $\mathrm{F}$ is in general identical to the path obtained independently after analysis of a simple model E. There is a catch, however. The fire resistance obtained after application of the model E proved to be significantly higher, than the one obtained after application of the model $\mathrm{F}$, this in turn means that such result should be treated as an undoubtedly overestimated value with respect to the real value of fire resistance, overestimating the safety level actually warranted to the user.

\section{CONCLUDING REMARKS}

The considerations presented here fit within the wider scope of research conducted by the authors and pertaining to the credibility of various computational models, differing in complexity, applied to estimate the fire resistance of steel hall bearing structures (Maslak et al, 2017, Wozniczka, 2019). In the juxtapositions presented here we intend to show, that application to that purpose of simple models based on the single transverse frame with appropriately modelled boundary conditions usually leads to overly optimistic estimates, more or less overestmating the level of safety warranted to the user. Of course, sufficiently careful modelling of support conditions and taking into account spatial nature of the potential deformations occurring in a frame exposed to fire action results in the fire resistance estimated on a model converging to the real value. The final result of the analysis is highly affected by the formal inclusion in the model of offsets induced by the way the purlins are supported on the rafter - this fact in 
a)

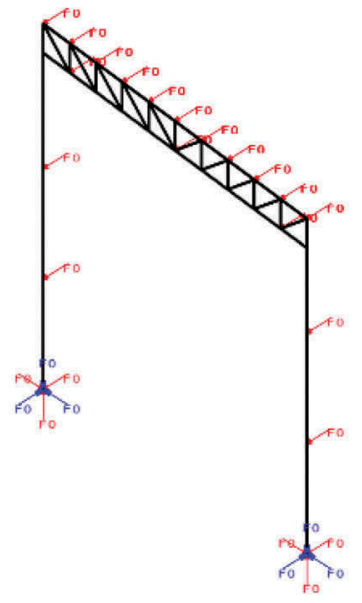

b)

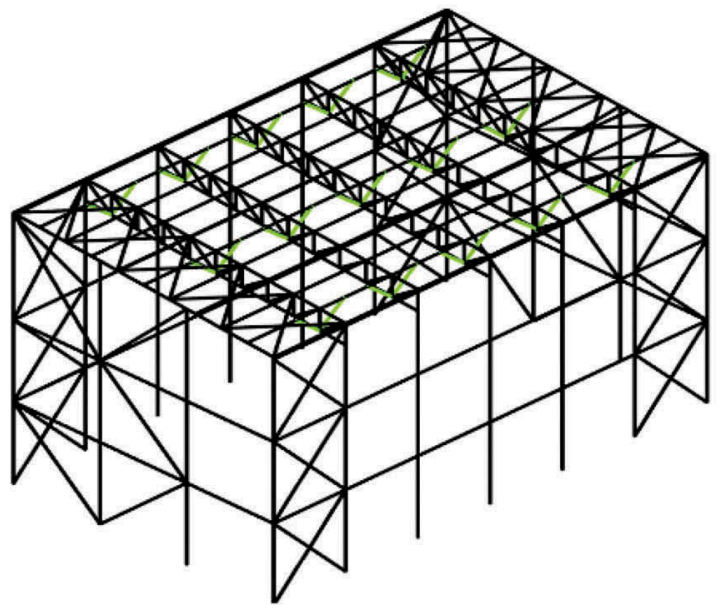

c)

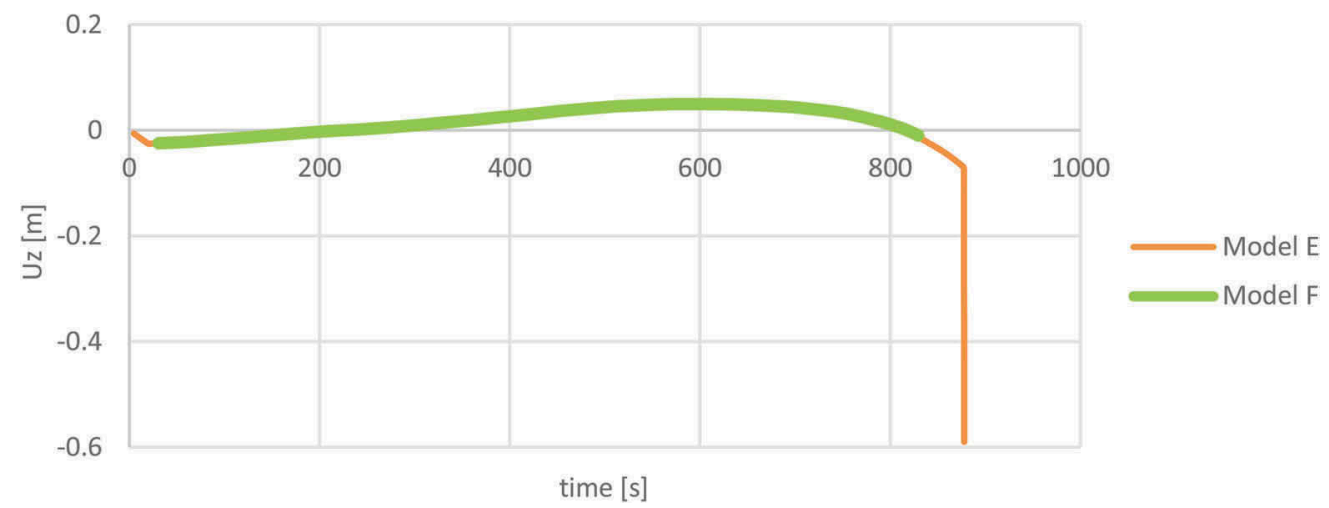

Figure 6. Verification of the credibility of results obtained after application of simple computational models, including: a) model of a single transverse frame (model E), b) 3D model taking into account the spatial interactions between structural components of the hall (model F), c) juxtaposition of the corresponding static equilibrium paths related to vertical displacements.

general is neglected in the analyses. The purlins induce an additional torsion in the rafter, and this in turn in the case of rafter rigidity decreased by the thermal action of fire may substantially accelerate its loss of stability.

In the computational models analyzed above the influence of potential substitute geometrical imperfections has been disregarded. The authors' research seems to indicate, that formal inclusion of these imperfections does not significantly affect the final estimate of fire resistance. Additional inclusion of wall girts in the analysis proved to be similarly insignificant. The destruction mode is the determining factor, and this is obviously determined by the support conditions defined in the model.

The question of selecting the optimum computational model for the analysis of fire resistance of a bar bearing structure in a steel hall, and especially sufficiently precise modelling of support conditions seems to be especially important if the complex 3D models, taking into account the 3D interactions between structural components, are selected for analysis (Jiang et al, 2018). One has to remember, that the rigidity of the structural components decreases with the increasing ambient temperature. This is accompanied by the increasing pliability of 
the joints, even those between columns and rafters. The joints, modelled as nominally rigid, under fire conditions with passing time become partially flexible, and this should be accounted for in the developed model. Research conducted so far seems to indicate as well, that the final fire resistance of a steel frame is highly affected by the behaviour of purlins stiffening the rafter (Obiala et al, 2010; Vassart et al. 2007). Those purlins, due to smaller cross section get heated much faster, than the rafter or columns of the transverse frame. Thus relatively early, at monotonous increase of temperature stop supporting the rafter and thus its increasing deflections, often determining the bearing capacity of the analyzed frame is no longer restrained in any way. Sufficiently precise modelling of the whole bracing system in the hall seems to be similarly important, as its influence changes during fire action.

\section{REFERENCES}

Franssen J.M. \& Gernay T. 2017. Modelling structures in fire with SAFIR: Theoretical background and capabilities. Journal of Structural Fire Engineering 8(3): 300-323.

Jiang J., Wang C., Lou G. \& Li G. 2018. Quantitative evaluation of progressive collapse process of steel portal frames in fire, Journal of Constructional Steel Research. 150: 277-287.

Maslak M., Tkaczyk A. \& Wozniczka P. 2017. The usability of a computational model of a single transverse frame in the fire resistance assessment of an entire steel hall. Safety \& Fire Technology. 45(1): 52-67.

Obiala R., Vassart O., Zhao B., Sakj M.S., de la Quintana J., Morente F., Fransen J.M. \& Lansival J.B. 2010. Fire safety of industrial halls. A valorization project. EUR 24222 EN Final Report. Luxembourg.

Vassart O., Brasseur M., Cajot L.G., Obiala R., Griffin A., Spasov Y., Renaud C., Zhao B., Arce C. \& de la Quintana J. 2007. Fire safety of industrial halls and low-rise buildings: realistic fire design, active safety measures, post-local failure simulation and performance based requirements. EUR $22568 \mathrm{EN}$ Final Report. Luxembourg.

Wozniczka P. 2019. Performance-based analysis of older-type large-space hall in fire. Archives of Civil Engineering. 65(1): 17-29.

Wozniczka P. 2020. Application of advanced fire resistance assessment method for industrial structures. Builder. 04 (273): 58-60 (in Polish). 